\title{
Haemolytic uraemic syndrome
}

Haemolytic uraemic syndrome and the related disorder thrombotic thrombocytopenic purpura have recently been the subject of much interest and research. ${ }^{1} \mathrm{~A}$ wide variety of phenomena have been observed and several pathogenetic mechanisms have been proposed, matched by a correspondingly diverse array of promising but unsubstantiated methods of treatment. Consideration of the heterogeneity of the clinical and laboratory findings suggests that it is improbable that haemolytic uraemic syndrome is a single disease entity and much of the present confusion is attributable to the multiplicity of pathogenetic mechanisms operative in different patients.

The triad of microangiopathic haemolytic anaemia, thrombocytopenia, and renal failure by which haemolytic uraemic syndrome is defined and its extension to include neurological involvement and fever (the pentad of thrombotic thrombocytopenic purpura) are clinical end points which may be reached by many different pathways. There may be a variety of initiating agents, perhaps viruses or bacteria, differing in their primary site of action which might be on the erythrocyte, platelet, endothelium, or coagulation system and there are presumably a range of host factors, congenital or acquired, affecting the vulnerability of these systems-yet the end result is a similar clinical picture. Evidence for such heterogeneity is to be found in epidemiological, histopathological, and pathophysiological studies which provide the basis for a tentative classification of the disorder.

\section{Epidemiology}

Haemolytic uraemic syndrome is predominantly a disease of infants and young children. Endemic foci occur particularly in Argentina and to a lesser extent in South Africa and the low countries. Elsewhere the disease occurs in mini epidemics such as the recent Midlands outbreak in the United Kingdom (unpublished data). It is more common in rural areas: only 6 of 75 children reported from the south east of England over a 12 year period lived in the London postal district. ${ }^{2}$ Analysis of this series suggested two distinct subgroups: an epidemic form occurring in younger children in the summer months, usually with an explosive onset of diarrhoea but with a good prognosis with supportive treatment alone; and in contrast, a sporadic form developing insidiously in older children with no seasonal variation in incidence and without a clear prodromal illness or with mild respiratory symptoms and having a much worse prognosis for renal function. ${ }^{2}$

Family studies support this distinction. ${ }^{3}$ Siblings who develop haemolytic uraemic syndrome within days or weeks of each other usually live in endemic areas and have the prodromal diarrhoea and good prognosis of the epidemic form. In the sporadic form of disease, however, which has been reported in 26 families, haemolytic uraemic syndrome develops in two to four siblings with more than a year separating the onset of disease in the individual cases. Most live in non-endemic areas and the prodromal illness is usually absent: the mortality in this group is high and they seem to inherit a predisposition to haemolytic uraemic syndrome as an autosomal recessive trait, though apparent dominant inheritance has also been reported.

It has been a natural supposition that the epidemic variety of haemolytic uraemic syndrome has an infective aetiology and it is well documented that shigella infection can result in an identical syndrome. ${ }^{4} \mathrm{~A}$ variety of other bacteria, viruses, and ricketsia have been implicated in individual cases but have not been isolated in subsequent studies. Reports of the syndrome after infection with neuraminidase producing organisms are of particular interest, for exposure of the ThomsenFriedenreich antigen consequent upon the stripping of sialic acid residues from erythrocytes by neuraminidase has been described in haemolytic uraemic syndrome. ${ }^{56} \mathrm{~A}$ recent report has also associated the syndrome with intestinal infection or colonisation with verotoxin producing Escherichia coli. ${ }^{7}$

Haemolytic uraemic syndrome in adults is associated with pregnancy, oral contraceptive use, and collagen vascular disorders. Severe hypertension from any cause may also produce microangiopathic haemolytic anaemia. In children, however, it is unusual to identify a systemic disorder underlying the condition.

\section{Histopathology}

As with other aspects of haemolytic uraemic syndrome there is also heterogeneity of histopathological appearances. ${ }^{89}$ The most common pattern is of a predominantly glomerular involvement with thrombotic microangiopathy. Endothelial cell swell- 
ing and separation from the basement membrane are prominent, together with widening of the subendothelial space. This appearance is typical of affected infants and is associated with a good prognosis - features which characterise the epidemic form of the syndrome. In contrast, in older patients with hypertension and a poor outcome for renal function, arteriolar rather than glomerular changes predominate with prominent intimal and sub-intimal oedema, proliferation, and necrosis. A third appearance occasionally encountered is acute cortical necrosis.

Are these histopathological subgroups indicative of different disease processes or do they represent variants of the same process? It is tempting to speculate that the patterns of predominantly glomerular or predominantly arteriolar involvement are the morphological counterparts of the epidemic and sporadic forms of the disease respectively.

\section{Pathogenesis}

Many of the early theories on haemolytic uraemic syndrome focussed on activation of the coagulation pathway as the primary event. The presence of fibrin in the renal lesions together with experimental evidence that microangiopathic haemolytic anaemia could be induced by activation of coagulation, enhanced by inhibition of fibrinolysis and diminished by anticoagulation, led to initial support for these hypotheses and enthusiasm for anticoagulation and fibrinolytic treatment. ${ }^{10}$ Subsequent studies, however, produced conflicting results and in general have failed to confirm the importance of activation of coagulation, as have the generally unsuccessful treatment trials of anticoagulation and fibrinolysis. Clotting studies are usually normal or only mildly abnormal and fibrinogen turnover is normal. ${ }^{11}$

As the apparent importance of coagulation has receded attention has shifted to the role of endothelium and platelets and their interaction in the pathogenesis of haemolytic uraemic syndrome. ${ }^{12}$ Studies of fibrinogen and plasminogen survival are normal whereas platelet turnover is noticeably increased in both adult ${ }^{11}$ and childhood disease. ${ }^{13}$ Furthermore platelets fail to aggregate normally and are depleted of both nucleotides and serotonin. ${ }^{14}$

Considerable advances in the understanding of how platelets are involved in haemolytic uraemic syndrome has come from the recognition of the role played by the arachidonic acid metabolites prostacyclin $\left(\mathrm{PGI}_{2}\right)$ and thromboxane $\left(\mathrm{TxA}_{2}\right)$ in regulating endothelial/platelet interactions. ${ }^{15}$ Arachidonic acid, the 20 carbon fatty acid precursor of the prostaglandins, is metabolised within the endothe- lial cell to $\mathrm{PGI}_{2}$, a vasodilator, and the most powerful inhibitor of platelet aggregation known. $\mathrm{PGI}_{2}$ is produced continually by endothelial cells and is a major factor in the thromboresistance of the endothelium. In contrast, arachidonic acid is metabolised within the platelet to $\mathrm{TxA}_{2}$, a powerful vasoconstrictor and platelet aggregating agent. A balance between the anti-aggregating effect of $\mathrm{PGI}_{2}$ and the pro-aggregatory effect of $\mathrm{TxA}_{2}$ is seen as being necessary for normal haemostasis. ${ }^{16}$

In 1978 Remuzzi suggested $\mathrm{PGI}_{2}$ deficiency as a cause of haemolytic uraemic syndrome and reported two patients whose blood vessels failed to produce $\mathrm{PGI}_{2}$ and whose plasma failed to support $\mathrm{PGI}_{2}$ production by endothelial tissue. ${ }^{17} 18$ Remuzzi postulated a deficiency in haemolytic uraemic syndrome plasma of a factor present in normal plasma necessary for $\mathrm{PGI}_{2}$ production. Support for the 'missing factor' hypothesis came from the finding that some patients responded to fresh plasma infusions. ${ }^{19}$ Evaluation of the therapeutic claims is difficult, however, in a disorder with a high rate of spontaneous recovery; moreover a few refractory patients have not responded to plasma but have improved instead with plasma exchange ${ }^{20}$ suggesting that removal of an inhibitor of $\mathrm{PGI}_{2}$ production may be involved. An inhibitor of $\mathrm{PGI}_{2}$ production has been detected in the plasma of patients with the sporadic form of disease by workers at two different centres $^{21} 22$ and preliminary characterisation suggests that it may be an abnormal lipid peroxide. ${ }^{21}$ Finally, excessively rapid degradation of $\mathrm{PGI}_{2}$ in haemolytic uraemic syndrome plasma has also been proposed. ${ }^{23}$ To add to the confusion, abnormality of $\mathrm{PGI}_{2}$ production has not been consistently detected in all patients with the syndrome and $\mathrm{PGI}_{2}$ infusions have had variable success only in treating the disorder. $^{24}$

In addition to these theories relating to $\mathrm{PGI}_{2}$ deficiency there are well documented reports of the presence of a platelet aggregating factor in the plasma of patients with thrombotic thrombocytopenic purpura. ${ }^{25}$ This factor induces aggregation of normal platelets and its activity can be inhibited by normal plasma suggesting that deficiency of a normal inhibitor of platelet aggregation may also be involved. The exact identity of this factor is not clear but a recent report suggests it may be an abnormal form of the factor VIII von Willebrand complex produced by endothelium. ${ }^{26}$ IgG immune complexes also aggregate platelets and may be involved in some of the patients with platelet aggregating factors. ${ }^{27}$ Patients with platelet aggregating factors seem to respond either to fresh plasma infusion or plasma exchange.

Theories favouring the endothelial cell as the 
primary site of damage have also been presented. Endothelial cytotoxic factors have been documented in several reports ${ }^{28}$ and in the epidemic form of haemolytic uraemic syndrome endotoxin, a well established endothelial toxin, has been identified in some cases. ${ }^{429}$

Although these conflicting theories on the pathogenesis of haemolytic uraemic syndrome offer exciting insights, they raise as many questions as they answer. Can we identify and therefore treat patients affected by each of these different mechanisms and do the established epidemiological and histological subgroups have pathophysiological counterparts?

On the basis of disturbed pathophysiology at least five subgroups of haemolytic uraemic syndrome can be identified at present:

(1) Deficiency of a plasma factor necessary for $\mathrm{PGI}_{2}$ production.

(2) Inhibition of $\mathrm{PGI}_{2}$ production.

(3) Excessively rapid degradation of $\mathrm{PGI}_{2}$.

(4) Presence of platelet aggregating factors.

(5) Presence of endothelial toxic factors.

In our experience the sporadic form of disease is often associated with mechanisms (1) to (4) and has a poor prognosis without vigorous treatment. A few patients with this form of haemolytic uraemic syndrome respond to fresh plasma infusions but plasmapheresis has been required to induce remission in most cases.

In contrast, the commoner epidemic form of disease is seldom associated with persistent $\mathrm{PGI}_{2}$ abnormality or platelet aggregating factors and spontaneous recovery with supportive treatment alone is the rule. While the pathophysiological mechanism in this form is still unknown, the histological and clinical features and the association with toxin producing coliform bacteria suggest an initial toxic insult to the endothelium.

Further dissection of the factors regulating the interaction between vascular endothelium and the circulating blood and their derangement in haemolytic uraemic syndrome will doubtless result in more precise definition of these subgroups and enable more specific treatment to be applied. Analysis of published results of treatment must take into account the type of patient treated. Haemolytic uraemic syndrome is a pathophysiological process that follows a variety of infective, immunological, or toxic insults superimposed on various inherent defects and an interdisciplinary study of the epidemiological, pathological, microbiological, and clinical features is required to complement the laboratory studies and unravel the full spectrum of the disorder.
ML was supported by the Kidney Research Aid Fund.

\section{References}

1 Fong JSC, de Chadarevian JP, Kaplan BS. Haemolytic uraemic syndrome: current concepts and management. Pediatr Clin North Am 1982;29:B35-56.

2 Trompeter RS, Schwartz R, Chantler C, et al. Haemolytic uraemic syndrome in childhood: analysis of prognostic features. Arch Dis Child 1983;58:101-5.

${ }^{3}$ Kaplan BS, Chesney RW, Drummond KN. Haemolytic uraemic syndrome in families. $N$ Engl $J$ Med 1975;292:1090-3.

${ }^{4}$ Koster F, Levin J, Walker L, et al. Haemolytic-uraemic syndrome after shigellosis: relation to endotoxemia and circulating immune complexes. $N$ Engl J Med 1978;298:927-33.

${ }^{5}$ Seger R, Joller P, Baerlocher K, et al. Haemolytic-uraemic syndrome associated with neuraminidase producing microorganisms: treatment by exchange transfusion. Helv Paediatr Acta 1980;35:359-67.

${ }^{6}$ Klein PJ, Bulla M, Newman RA, et al. Thomsen Friedenreich antigen in haemolytic uraemic syndrome. Lancet 1977;ii:1024-5.

7 Karmali MA, Steele BT, Petric M, Lim C. Sporadic cases of haemolytic-uraemic syndrome associated with faecal cytotoxin and cytotoxin producing Escherichia coli in stools. Lancet 1983;i:619-20.

${ }^{8}$ Habib R, Levy M, Gagnadoux MF, Broyer M. Prognosis of the haemolytic-uraemic syndrome in children. In: Morton, Maxwell, eds. Advances in nephrology. Chicago, London: Year Book Medical Publishers, 1982:99-128.

${ }^{9}$ Kanfer A. Morel-Maroger L, Solez K, Sraer JD, Richet G. In: Remuzzi G, Mecca G, De Gaetano G, eds. The value of renal biopsy in hemolytic uremic syndrome in adults. New York: Raven Press, 1980:399-406.

${ }^{10}$ Brain MC. The haemolytic-uraemic syndrome. Semin Hematol 1969;6:162-80.

1 George CRP, Slichter SJ, Quadracci LJ, Striker GE, Harker LA. A kinetic evaluation of hemostasis in renal disease. N Engl J Med 1974;291:1111-5.

12 Anonymous. Platelets, endothelium and renal disease (editorial). Lancet 1979;ii:890-2.

${ }^{13}$ Katz J, Krawitz S, Sacks PV, et al. Platelet, erythrocyte and fibrinogen kinetics in the hemolytic uremic syndrome of infancy. $J$ Pediatr 1973;83:739-48.

14 Fong JSC, Kaplan BS. Impairment of platelet aggregation in hemolytic uremic syndrome: evidence for platelet 'exhaustion'. Blood 1982;60:564-70.

15 Moncada S, Vane JR. Prostacyclin: its biosynthesis, actions and clinical potential. Phil Trans R Soc London /Bioll 1981:294: 305-29.

16 Moncada S, Vane JR. Prostacyclin, platelet aggregation and thrombosis. In: De Gaetano G, Garattini S, eds. Platelets: $a$ multi-disciplinary approach. New York: Raven Press, 1978: 239-58.

17 Remuzzi G, Marchesi D, Mecca G, et al. Haemolytic-uraemic syndrome: deficiency of plasma factor(s) regulating prostacyclin activity? Lancet 1978;ii:871-2.

${ }^{18}$ Remuzzi G, Mecca G, Livio M, et al. Prostacyclin generation by cultured endothelial cells in haemolytic-uraemic syndrome. Lancet 1980;i:656-7.

${ }^{19}$ Misiani R, Appiani AC, Edeforti A, et al. Haemolytic uraemic syndrome: therapeutic effect of plasma infusion. $\mathrm{Br}$ Med $\mathrm{J}$ 1982;285:1304-6.

${ }^{20}$ Bukowski RM, King JW, Hewlett JS. Plasmapheresis in the treatment of thrombotic thrombocytopenic purpura. Blood 1977;50:413-7.

${ }^{21}$ Levin M, Elkon KB, Buckle AM, et al. Inhibitor of prostacyclin production in sporadic haemolytic uraemic syndrome. Arch Dis Child 1983;58:703-8.

${ }^{22}$ Stuart MJ, Spitzer RE, Coppe D. Abnormal platelet and 
vascular prostaglandin synthesis in an infant with the hemolytic uremic syndrome. Pediatrics 1983;71:120-4.

${ }^{23}$ Chen YC. Hall ER. McLeod B. Wu KK. Accelerated prostacyclin degradation in thrombotic thrombocytopenic purpura. Lancet 1981;ii:267-9.

24 Defreyn G, Proesmans W, Machin SJ, Lemmens F. Vermylen J. Abnormal prostacyclin metabolism in the hemolytic uremic syndrome: equivocal effects of prostacylin infusions. Clin Nephrol 1982;18:43-9.

25 Lian ECY. Harkness DR, Byrnes JJ, Wallach H. Nunez R. Presence of a platelet aggregating factor in the plasma of patients with thrombotic thrombocytopenic purpura (TTP) and its inhibition by normal plasma. Blood 1979:53:333-8.

26 Moake JL, Rudy CK. Troll JH, et al. Unusually large plasma factor VIII von Willebrand factor multimers in chronic relapsing thrombotic thrombocytopenic purpura. $N$ Engl $J$ Med 1982;307:1432-5.

27 Sacher RA, Phillips TM, Shashaty GG, Jacobsen RJ, Rath CE, Lewis MG. Demonstration of immune complexes in thrombotic thrombocytopenic purpura and effect of exchange transfusion. Scand J Haematol 1980;24:373-80.

2x Burns ER, Zucker-Franklin D. Pathologic effects of plasma from patients with thrombotic thrombocytopenic purpura on platelets and cultured vascular endothelial cells. Blood 1982;60:1030-7.

29 Semaro N. Interactions of platelets, leucocytes and endothelium with bacterial endotoxins: possible relevance in kidney disorders. In: Remuzzi G, Mecca G, De Gactano G, eds. Hemostasis, prostaglandins and renal disease. New York: Raven Press, 1980:99-116.

\section{Levin and T M BarRatT Department of Paediatric Nephrology, Institute of Child Health, 30 Guilford Street, London WCIN IEH}

